\title{
Flux Flow, Pinning, and Resistive Behavior in Superconducting Networks
}

\author{
Annual Progress Report
}

for the period May 1, 1992 to April 30, 1993

\author{
Stephen Teitel
}

Department of Physics and Astronomy

University of Rochester

Rochester, New York 14627

October 1992

Prepared for

THE U. S. DEPARTMENT OF ENERGY

GRANT NO. DE-FG02-89ER 14017

\section{DISCLAIMER}

\footnotetext{
This report was prepared as an account of work sponsored by an agency of the United States Government. Neither the United States Government nor any agency thereof, nor any of their employees, makes any warranty, express or implier, or assumes any legal liability or responsibility for the accuracy, completeness, or usefulness of any information, apparatus, product, or process disclosed, or represents that its use would not infringe frivately owned rights. Reference herein to any specific commercial product, process, or service by trade name, trademark, manufacturer, or otherwise does not necessarily constitute or imply its endorsement, recommendation, or favoring by the United States Government or any agency thereof. The views and opinions of authors expressed herein do not necessarily state or reflect those of the United States Government or any agency thereof.
}

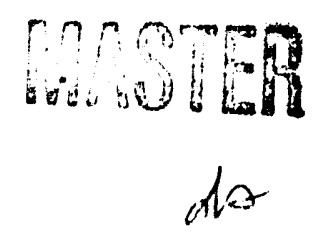




\begin{abstract}
We have studied the behavior of fluctuation effects in superconducting systems using numerical simulations of $X Y$ and Coulomb gas models. The fluctuation of vortex lines in high temperature superconductors, in the presence of an external magnetic field, has been studied using a three dimensional $X Y$ model. We have carried out new simulations on a more dilute vortex line model than we considered previously, and find surprising evidence that the process of vortex line lattice melting occurs as two distinct phase transitions. As the vortex line lattice is heated, it melts first into a vortex line liquid of straight unentangled lines, having superconducting properties in the direction along the lines (parallel to the external field), but not in directions transverse to the lines. At a higher temperature, the thermal excitation of closed vortex line loops links all the lines together, leading to a tangled vortex line liquid, with completely normal metal properties in all directions. We have also continued simulations of the two dimensional Coulomb gas, as a model for vortex fluctuations in two dimensional arrays of Josephson junctions. We find phase transitions which do not have the critical beliavior expected from standard symmetry analyses.
\end{abstract}

\title{
NOTICE
}

This report was prepared as an account of work sponsored by the United States Government. Neither the United States nor the Department of Energy, nor any of their employees, nor any of their contractors, subcontractors, or their employees, makes any warranty, express or implied, or assumes any legal liability or responsibility for the accuracy, completeness, or usefulness of any information, apparatus, product or process disclosed or represents that its use would not infringe privately-owned rights. 


\section{Summary of Progress for the Period 5/1/92-4/30/93}

Research has continued, modeling fluctuation effects in superconducting systems, in terms of well defined statistical models which may be numerically simulated. These models are the uniformly frustrated $X Y$ model, in which the angle of planar spins located at the nodes of a network, model the phase of the superconducting wavefunction as it varies in space; and the Coulomb gas model, in which Coulomb interacting "charges" model the interaction between vortices in the phase of the superconducting wavefunction, in two dimensional superconducting Josephson junction arrays. In this period, we have focused on studying the fluctuations of magnetic field induced vortex lines in the three dimensional $X Y$ model, as a model for behavior in high temperature superconductors. We have continued to study the phase transitions of the Coulomb gas model, considering specific cases which are experimentally realizable in Josephson arrays. We have also extended these Coulomb gas studies to include the steady state response to a driving field, as a model for flux flow resistance, and we are comparing these results against proposed scaling laws. Some preliminary simulations have been carried out to study the effects of positional disorder and gauge disorder in two dimensional superconductors. Details of these efforts are given below.

\section{A. Vortex Line Fluctuations in High Temperature Superconductors}

We have continued our simulations of vortex line fluctuations in high temperature superconductors, using the three dimensional $X Y$ model. Experimental evidence continues to show that such vortex line fluctuations play a key role in determining the resistive behavior of these materials in the presence of an applied magnetic field. Our efforts have been in improving upon simplifications made in our earlier work, in order to get a more realistic model of the high $T_{C}$ materials.

One approximation in our numerical approach has been the replacement of continuous space with a finite numerical mesh of discrete points. This is most valid when the spacing between points on the numerical mesh is small compared to other relevant physical lengths, in particular the spacing between the magnetic ficld induced vortex lines, $a_{\nu}$. Our earlier work involved a relatively high density of vortex lines, with $a_{\nu}=\sqrt{5}$, as measured in units of the numerical mesh lattice constant (density of lines is $f=1 / 5$ ). In collaboration with Dr. YingHong Li (formerly a posidoc supported by this grant, then a postdoc at the Institute of Theoretical Physics in Utrecht, currently a visiting rescarch associate back in Rochester) I have carried out new simulations on a more dilute system with $a_{\nu}=5$ (density $f=1 / 25$ ). This value of $f$ was chosen so as to increase the line spacing, while still giving a large number of lines contained within a system of computational size. 
These new simulations confirmed our previous results, indicating that vortex lines easily cut through each other once the' start to entangle. Hence vortex line entanglement does not pose topological constraints against vortex line motion. However we also found surprising new results, indicating that the vortex line lattice melting transition, accompanying the transition from the superconducting to normal state, occurs as a distinct two step process. As the vortex line lattice is heated, there is first a transition to a line liquid of approximately straight and unentangled lines. This line liquid is characterized by having phase coherence (and hence being superconducting) along the direction of the applied magnetic field (corresponding to the direction of the straight vortex lines), while being phase incoherent (and hence not superconducting) in the perpendicular directions. Upon heating further, there is a second transition where the phase coherence in the magnetic field direction is also lost, and the system become completely normal. This higher transition is mediated by an entangling of the vortex lines, due to the thermal excitation of many closed vortex line loops, which intersect and link up the field induced vortex lines. We are currently exploring the possibility that this higher transition might be related to the low temperature "shoulder" observed in resistivity measurements in very pure samples of $Y B C O$.

In these new simulations, we have repeated the analysis of the vortex line structure function, as in our earlier work (publication \#2, Section III). We have extracted from our data the effective, wave vector dependent, line tension of the vortex lines, and found reasonable agreement with the form predicted by earlier elastic theories. The results of these new simulations have been accepted for publication in Physical Review B (publication \#4, Section III).

We have also, most recently, restudied the above model, but varying the size of the numerical mesh in order to see if any of the new results discussed above were merely artifacts of looking at too small a system. Comparing results from meshes of sizes $25 \times 25 \times L$, with $L=25,50$, and 100 along the direction of the magnetic field, we find no evidence that any of our conclusions change. In particular, we continue to find two distinct and well separated transitions associated with the vortex line lattice melting. A manuscript describing these results is in preparation.

A second approximation made in our work, has been to assume that the magnetic field present in the system is completely uniform. This is good for describing behavior on length scales small compared to the magnetic penetration length $\lambda$, and hence good for describing vortex line lattice melting when $\lambda \gg a_{v}$ the spacing between lines. Since $a_{v}$ decreases as the magnetic field increases, this is good in the high field limit. In order to study the low field limit, or to compute long length scale compressibilities even in the high field limit, it becomes necessary to include the spatial variations, and thermal fluctuations of the 
magnetic field. In collaboration with my graduate student Tao Chen, I am extending our simulations to include such local variations in the magnetic field, keeping the average field constant. Such simulations are similar in character to those associated with "lattice gauge theory" of high energy physics, and greatly increase the complexity of the problem and the computational resources needed. Although progress here has been initially slow, we believe we have recently succeeded in working out the technical problems, and have begun simulations. Our preliminary results suggest that the two step vortex line lattice melting transition found in the high field approximation, remains even in the low field limit. Much further work remains to be done, and our investigations of this more complex model will form a large part of our work in the next budget period.

\section{B. Equilibrium Phase Transitions in the Two Dimensional Coulomb Gas}

In collaboration with my graduate student, Jong-Rim Lee, I have continued study of equilibrium phase transitions in two dimensional Coulomb gas systems, which model the melting transitions of $2 d$ point vortex lattices in thin film superconductors or periodic arrays of Josephson junctions. We have extended earlier work on the integer Coulomb gas on square and triangular lattices, as well as carried out new studies on the $f=1 / 2$ and $f=1 / 3$ fractional Coulomb gases on the triangular lattice. These later two cases correspond to the $2 d$ honeycomb Josephson junction array with $1 / 2$ and 1/3 flux quanta of magnetic field per unit cell. The transition in the $f=1 / 3$ case is expected by symmetry analysis to be in the universality class of the 3-state Pott's model. However, as we found with other cases, the observed transition has apparently different critical behavior. The results have been published in Physical Review B (publication \#3, Section III)

Most recently, Mr. Lee has re-examined the simplest of the fractional Coulomb gas models, the $f=1 / 2$ model on a square lattice, which models a square Josephson array with $1 / 2$ flux quanta per cell. Recent results by Kosterlitz et al., and by José et al., in the $X Y$ model representation, argued that the vortex lattice melting transition in this model is not Ising-like, as had been suggested by earlier works. Mr. Lee's work, using completely different methods of finite-size-scaling analysis, confirms the non-Ising nature of this melting transition. However his simulations go beyond these new works, finding clear evidence that the metal-insulator transition of this Coulomb gas (corresponding to the loss of superconductivity in the corresponding Josephson array) occurs at a temperature distinctly lower than the vortex lattice melting transition, and does not have the universal behavior as in the expected KosterlitzThouless transition. A manuscript describing these results is in preparation. 


\section{Steady State Vortex Flow in the Driven Two Dimensional Coulomb Gas}

The behavior of vortices in a two dimensional superconductor, in the presence of an externally applied d.c. current, can be mapped onto the behavior of Coulomb interacting charges in the presence of an applied uniform electric field. The resulting flux flow voltage in the superconductor, corresponds to the charge current in the Coulomb model. In collaboration with J.-R. Lee, I have used this correspondence to study the steady state dynamic behavior of flux flow in two dimensional Josephson junction arrays, using driven diffusion Monte Carlo simulations.

Fisher, Fisher and Huse have proposed a general scaling law for the d.c. current-voltage characteristics of any second order superconducting transition. In the limit of asymptotically small current, this scaling law gives linear (Ohmic) behavior above $T_{c}$, nonlinear behavior below $T_{C}$, and a power law behavior $V \sim I^{\gamma}$ exactly at $T_{c}$. Although this scaling law has been used to analyze experimental and numerical simulation data for the complicated "vortex glass" problem, no systematic study of its validity had yet been carried out.

We have therefore studied the simplest case possible, of a disorder free $2 d$ superconductor when there is no applied magnetic field. Here one expects a Kosterlitz-Thouless phase transition, with a power law relation between voltage and current at $T_{c}, V \sim I$, with the value $\gamma=3$ predicted by the vortex pair unbinding theory. By carrying out simulations exactly at $T_{c}$, for systems of various size $L$, we make a systematic finite-size-scaling test of the proposed scaling relation. We find our results consistent with the general scaling law within our statistical errors. However it appears that the current scale at which one enters the asymptotic power law region, occurs at a smaller current than one would expect from the dimensional analysis implicit in the general scaling law. A manuscript describing these results is in preparation.

\section{Disordered Two Dimensional Superconductors}

In collaboration with Prof. Jan Tobochnik of Kalamazoo College, I have been investigating the equilibrium behavior of positionally disordered, and gauge disordered, two dimensional Josephson junction arrays, using the $X Y$ model description. In contrast to earlier work on these problems, we are using a new test for phase coherence, which was developed in my earlier work on a bond diluted array (publication \#1, Section III). Our results are still preliminary. 


\section{Research Plans for the Next Budget Period (5/1/93 - 4/30/94)}

We will continue work on the above tupics, as well as attempt new simulations on vortex line flow in an applied d.c. current in a three dimensional model superconductor, as discussed in greater detail below.

\section{A. Vortex Line Fluctuations in High Temperature Superconductors}

We will continue our simulations of the three dimensional frustrated $X Y$ model, including fluctuations of the local magnetic field, in order to model the more realistic case of vortex lines interacting with a finite interaction length (the magnetic penetration length) $\lambda$. We will consider the cases $\lambda \sim a_{\nu}$, as well as the weakly interacting case $\lambda \ll a_{\nu}$ (where $a_{\nu}$ is the average spacing between vortex lines) in order to investigate any changes in behavior from that seen previously seen in our $\lambda \rightarrow \infty$ approximation. We will also use the results of this finite $\lambda$ model, to compute the long wavelength vortex line compression modulus, which can be related to the diamagnetic susceptibility of the superconductor. This would be the first such numerical calculation of the diamagnetic response in such a model pure superconductor, and is important since a characteristic experimental feature of the high $T_{c}$ superconductors is the observation that the onset of significant diamagnetism occurs at a temperature substantially higher than the resistive transition temperature.

\section{B. Equilibrium Phase Transitions in the Two Dimensional Coulomb Gas}

Recently, Moore has argued that for a vortex lattice in a pure (pinning free) two dimensional superconductor, the long wavelength elastic shear modes of the lattice are sufficient to destroy phase coherence of the superconducting wavefunction (and hence superconductivity) at all finite $T \neq 0$. We have been unable to investigate this effect in our lattice Coulomb gas models, as the restriction that the charges (vortices) sit only on the sites of a discrete numerical mesh, produces an effective periodic pinning potential that suppresses the long wavelength shear excitations. We therefore will attempt a numerical simulation of the Coulomb gas in a uniform continuum, to see if we recover Moore's predictions when pinning is completely removed.

\section{Steady State Vortex Flow in the Driven Two Dimensional Coulomb Gas}

We will continue our driven diffusion simulations of the two dimensional Coulomb gas as a model for flux flow in Josephson junction arrays. We will extend our work to consider the case of the $f=1 / 2$ fractional model (one half flux quantum per unit cell, see Section I.B above), to see if the general scaling relation holds in this case, and to see if these dynamic 
simulations shed any light on the nature of the phase transition in this system, which is still incompletely understood. We will also consider the $2 d$ driven diffusion Coulomb gas in a random field, as a model for flux flow with random vortex pinning.

\section{Steady State Flux Flow in Three Dimensional Superconductors}

So far, we have modeled the fluctuations of a bulk superconductor in terms of a three dimensional $X Y$ model. It is also possible to directly model the interaction between fluctuating vortex lines in this system, in a similar way as Coulomb charges model point vortices in a $2 d$ (thin film, Josephson array) superconductor. Such simulations have already been carried out to study equilibrium properties by Carneiro et al. We hope to extend this work to consider the steady state dynamic behavior in the presence of an applied d.c. current, using drift diffusion Monte Carlo simulations analogous to those we are carrying out on the $2 d$ Coulomb gas (Section II.C). We will look for features in the computed resistivity that reflect the two step vortex lattice melting transition which we have found in our equilibrium simulations. Such an analysis should help to make greater connection between the behaviors we have found in our models, and experimental measurements on the high $T_{c}$ materials. 
III. Publications Acknowledging DOE Support, for the Current Period (5/1/92 - 4/30/93)

1. The Effect of Random Pinning Sites on Behavior in Josephson Junction Arrays, Ying-Hong Li and S. Teitel, Phys. Rev. Letts. 67, 2894 (1991)

2. The Structure of a Dense Vortex Line Liquid in a Model High $T_{c}$ Superconductor, Ying-Hong Li and S. Teitel, Phys. Rev. B 45, 5718 (1992)

3. Phase Transitions in Classical Two Dimensional Lattice Coulomb Gases, Jong-Rim Lee and S. Teitel, Phys. Rev. B 46, 3247 (1992)

4. Vortex-Line Fluctuations in Mcdel High $T_{c}$ Superconductors, Ying-Hong Li and S. Teitel, Phys. Rev. B, accepted for publication

IV. Seminar and Conference Presentations, for the Current Period (5/1/92 - 4/30/93)

Vortex-Line Fluctuations in High Temperature Superconductors, presented at:

1. Tenth Symposium on Energy Engineering Sciences, Argonne, IL, 5/11/92

2. Workshop on: "Inertial Sensors: An Application of the Physics of Superconductors," sponsored by the Office of Naval Research (invited talk), Vail, CO, 6/10/92

3. Condensed Matter Seminar, Weizmann Institute of Science, Rehovot, Israel 7/14/92

4. Condensed Matter Seminar, Tel Aviv University, Tel Aviv, Israel, 8/19/92

5. Condensed Matter Seminar. Technion, Haifa, Israel, $8 / 20 / 92$ 


\section{Personnel Involved With The Project}

Dr. Stephen Teitel, Associate Professor (PI)

$50 \%$ of my effort during the academic year, $100 \%$ effort during the summer, has been devoted to this project during the entire period of the grant.

Jong-Rim Lee, 6 th year graduate student

Mr. Lee has been a graduate student with me for the last four years. During this time, he has been supported by this grant, devoting $100 \%$ of his effort to this project, primarily on the Coulomb gas projects described above. It is anticipated that Mr. Lee will complete his Ph.D. work this summer.

Tao Chen, 4th year graduate student

Mr. Chen has been a graduate student with me for the last two years. This last year he has been supported by this grant, devoting $100 \%$ of his effort to this project, particularly in extending the modeling of vortex line fluctuations to include the effects of magnetic field fluctuations. Mr. Chen will continue to complete his Ph.D. thesis working on topics related to this project.

Marcel Franz, 3rd year graduate student

Mr. Franz will join this program next semester (Spring '93), and will be assigned to one of the new projects, probably that discussed in Section II.B. This year he has been supported as a teaching assistant by the Department. It is expected that he will be supported as a research assistant on this grant starting Sept. '93, and will continue to complete his Ph.D. thesis on topics related to this project.

VI. Budgetary Information for the Current Period (5/1/92 - 4/30/93)

No unexpended balance is anticipated at the end of the current funding period. 

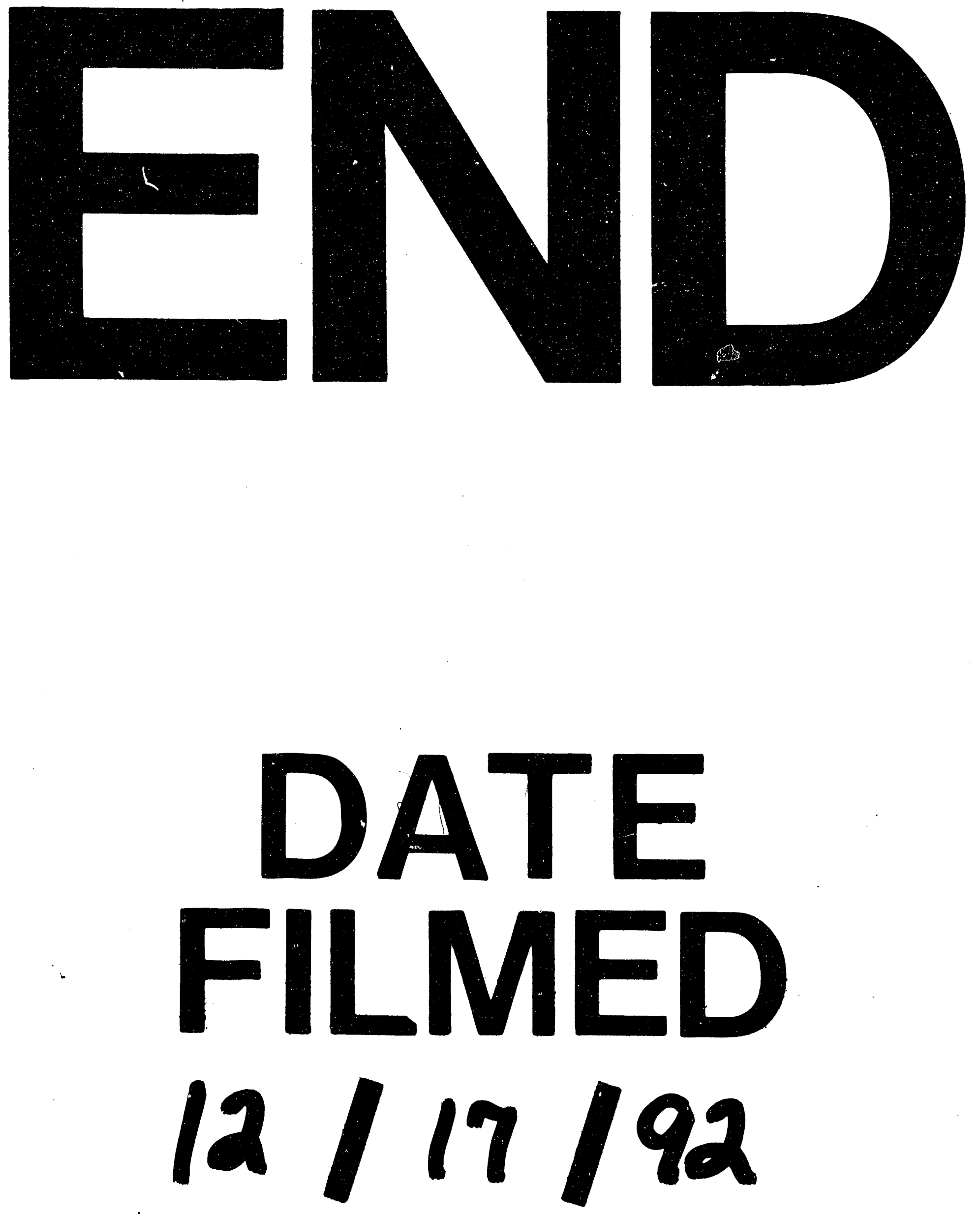


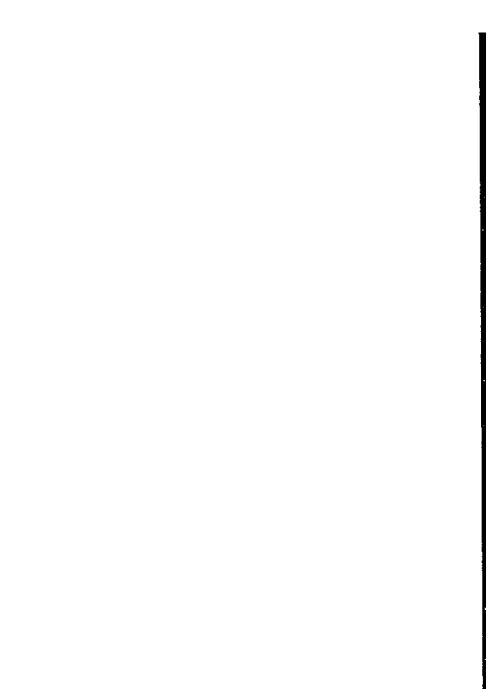

